\title{
Future Accelerator Challenges
}

\author{
Attilio Andreazza*t \\ Universtità degli Studi di Milano and INFN Sezione di Milano \\ E-mail: attilio.andreazza@mi.infn.it
}

\begin{abstract}
Improvements in our knowledge of fundamental interactions are expected in the next years from the upgrade of existing accelerators or new machines under design. The full exploitation of the physics potential of these machines will require new and more performant detectors. The next decade will be characterised by the high-luminosity LHC upgrade. New solutions in particular for the tracking system and the forward detector are beng proposed. A major upgrade will be on the trigger system aiming to exploit the maximum granularity and tracking information already at the first level. But other projects are also emerging, as challenging examples are the concept of an extreme flavour physics experiment and the fine granularity and high resolution detectors for future lepton colliders.
\end{abstract}

INFN Workshop on Future Detectors for HL-LHC

16-18 December, 2015

Aula Magna della Cavallerizza Reale, Torino, Italy

\footnotetext{
${ }^{*}$ Speaker.

${ }^{\dagger}$ for INFN Commissione Scientifica Nazionale 1
} 


\section{Introduction}

New accelerators, or significant upgrades of existing ones, bring also new generation of detectors to improve the physics reach of the experiments.

The current situation in particle physics presents an extremely interesting duality. On one side there is a successful Standard Model, whose particle set has been completed with the discovery of the Higgs boson [1] and which has passed all precision tests up to now. At the same time there is evidence for the need of new particles and interactions: the Standard Model cannot describe the current universe, since it does not explain the presence for dark matter and the particle-antiparticle asymmetry.

This situation is extremely challenging, because there are little clues about at which scale or in which processes new physics can be observed. Therefore a wide range of experiments need to be planned to cover any possible scenario. The INFN CSN1 has produced a white paper [2] showing a rich set of ideas about how to pursue the exploration of fundamental interactions at present and future accelerator facilities.

In order to concretise into proposals for new experiments, most of these ideas require the development of new techniques or to push performance beyond the state of art of detector technology. In this presentation, after a quick review of the physics motivations presented in [2], some selected topics are presented, with emphasis to the next immediate challenge: the High Luminosity upgrade of the Large Hadron Collider (HL-LHC) [3]. More details and references can be found in specific contributions to this workshop $[4,5,6,7,8,9,10]$.

\section{Physics challenges at accelerators}

As mentioned in the introduction, there are many complementary ways to search for processes beyond the Standard Model.

A first step towards the exploration of new physics, is the precision measurement of Standard Model processes. Sectors of particular interests are the properties of the heaviest particles, the Higgs boson and the top quark, and the study of the self-interaction of gauge bosons via di-boson scattering. A significant improvement in precision will come from the large amount of data that will be available from the $3 \mathrm{ab}^{-1}$ integrated luminosity expected from the HL-LHC. A further major step can be performed with a lepton collider covering the centre-of-mass energy region from the Higgs threshold production to the top quark pair threshold. As an example, fig. 1(a) shows the level of precision reachable by HL-LHC and electron-positron colliders on the Higgs boson couplings.

The direct search for new particles has two frontiers to explore. On the high mass region, after the running of HL-LHC at $14 \mathrm{TeV}$, new machines are definitely needed, either a hadron collider at $\sqrt{s} \approx 100 \mathrm{TeV}$, or a lepton collider with $\sqrt{s} \geq 1 \mathrm{TeV}$. An alternative scenario is the low-mass and low-coupling regime, looking for particles that has escaped detection till now due to their extremely weak interaction. The challenge in these kind of experiments (among which SHIP [12] and PADME [13] are notable examples) are the large beam intensities and the background suppression. Figure 1(b) illustrates on a particular model, in which the standard muon neutrino mixes with a heavy neutral lepton (HNL), how different machines with different particles, luminosities 
and energy scales are complementary in covering different regions of the parameter space (in this case the mass of the HNL and the square if its mixing term with the neutrino).

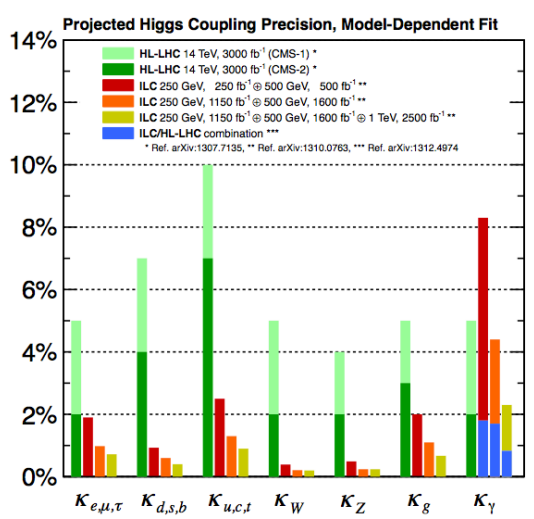

(a)

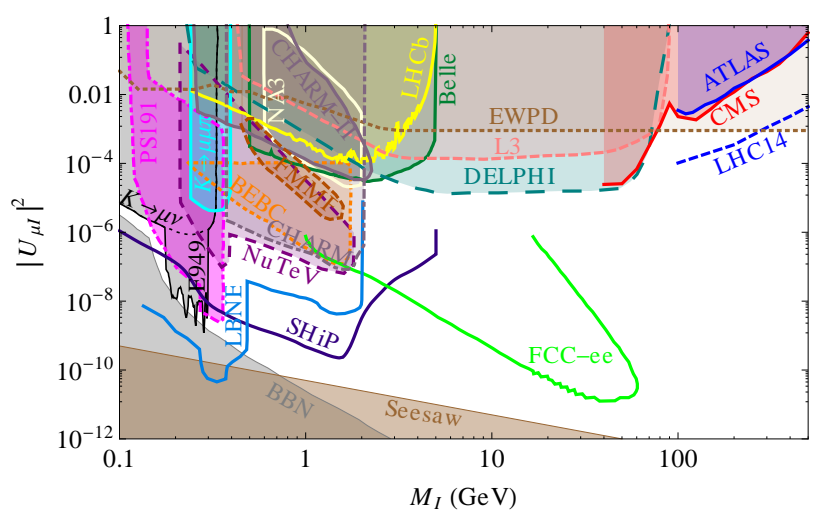

(b)

Figure 1: (a) Model-Dependent projected precision on Higgs-boson coupling scaling factors. Green bands refer to HL-LHC projections; blue bands refer to combinations of ILC and HL-LHC outputs [2, 11]. (b) Limits on the mixing between the muon neutrino and a single heavy neutral lepton in the mass range 0.1$500 \mathrm{GeV}[12]$.

A vast landscape of experiments, either running or in the construction phase, will study in the next decade flavour physics at an unprecedented level of precision. Just to name a few experiments in which INFN is involved, lepton flavour violation will be probed at MEG and Mu2e, rare or forbidden decays of kaons are studied at KLOE and NA62, heavy flavours at LHCb, BES III and BELLE II, together with precision measurements of CKM matrix elements. Perspectives beyond this program require a technological step to be able to manage enormous data samples $\left(10^{14-15}\right.$ heavy quarks decays) and beam intensities ( $\left.>10^{19} \mathrm{pot} / \mathrm{yr}\right)$.

With such a wide range of challenges, it is a must for the particle physics community at accelerators to develop plans for the full exploitation of the HL-LHC, which is the first priority in the European Strategy for Particle Physics [14]. At the same time concrete proposal are expected for new experiments in the next few years: their prioritisation will depend on the output of the Run-2 of the LHC and the status of R\&D on magnets, accelerating techniques, detectors, data acquisition and analysis methods.

\section{Detectors for the High-Luminosity LHC}

The future schedule of the LHC and of the HL-LHC upgrade is sketched in fig. 2. Currently the HL-LHC is expected to deliver, starting from 2026, high intensity beams providing $5 \times 10^{34} \mathrm{~cm}^{-2} \mathrm{~s}^{-1}$ instantaneous luminosity. At $25 \mathrm{~ns}$ bunch spacing, there will be on average $140 p-p$ interactions per bunch crossing, with an interaction density of $1.25 \mathrm{event} / \mathrm{mm}$ [3].

This change in operation condition requires a major upgrade of the general purpose experiments ATLAS and CMS $[15,16]$. The most relevant features of these upgrades are the complete replacement of the tracking system, the strengthening of the forward region detectors and a mod- 


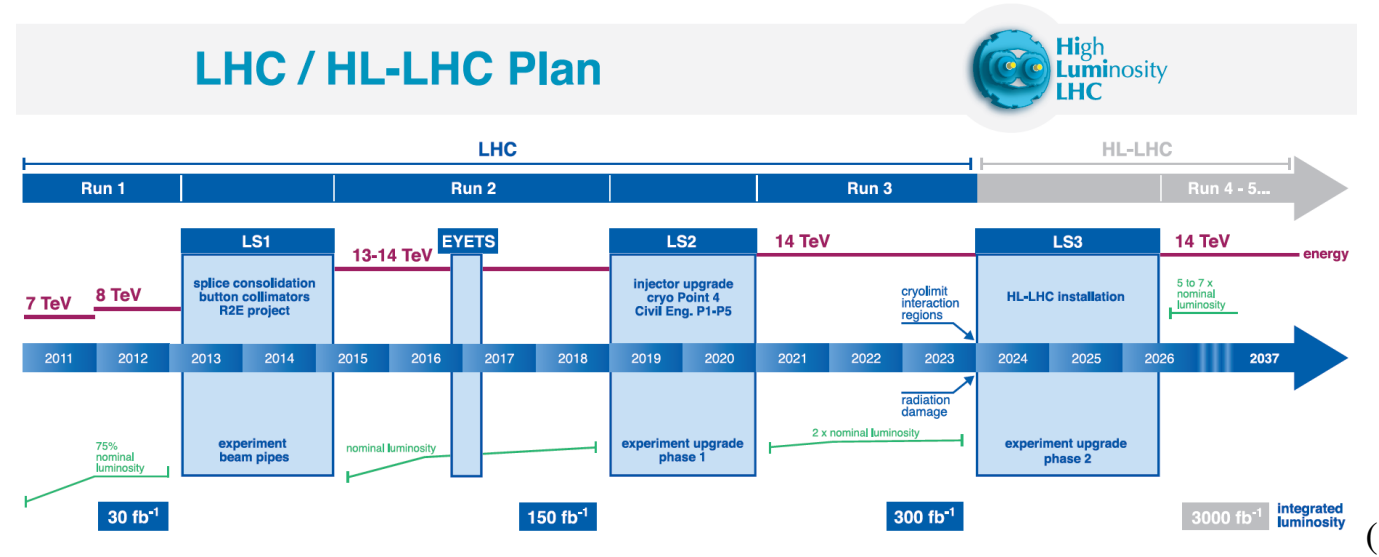

Figure 2: Schedule of the LHC and of its High Luminosity upgrade.

ification of the trigger components and strategy to maintain high efficiency despite the increased pile-up level.

The tracking systems have to fulfil tighter requirement in term of radiation hardness, with the innermost layer having to face a total fluence of $2 \times 10^{16}$ particles $/ \mathrm{cm}^{2}$. The layouts of the ATLAS and CMS upgraded trackers are drawn in fig. 3. The higher particle density requires a finer granularity than the current LHC conditions: silicon pixel detectors will implement a segmentation of $50 \times 50$ or $25 \times 100 \mu \mathrm{m}^{2}$ and will be adopted up to a larger radius. They will also be exploited to increase the tracking system coverage down to a pseudorapidity $|\eta|<4$. New sensor technologies are being developed to cope with the radiation requirements and to reduce the costs of the wide silicon surfaces [4]. To cope with the high data rate, the hybrid pixel solution is currently the only option, with a worldwide R\&D effort on the $65 \mathrm{~nm}$ technology node for the fabrication of the front-end electronics [5].

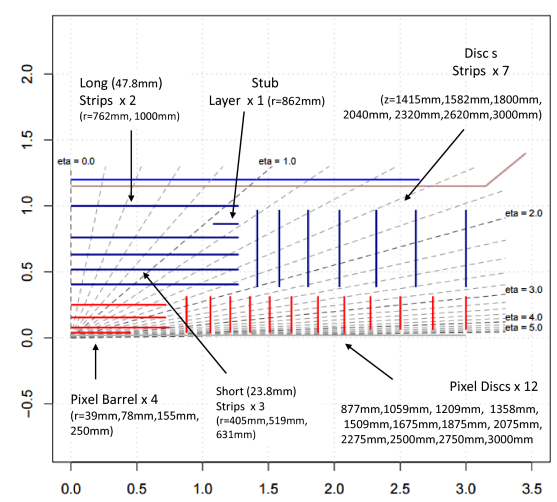

(a)

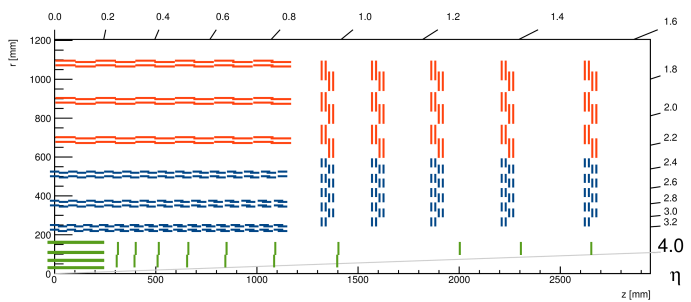

(b)

Figure 3: Layout of the upgraded inner tracking systems for the HL-LHC: (a) ATLAS: pixel detector in red, strip detectorc in blue [15]; (b) CMS: pixel detector in green, macro pixel+strip layers in blue, double strip layers in red. [16]. 
The other region where pile-up effects will have the most impact is the one covered by the forward detectors. Both experiments are planning upgrades of their forward calorimetry in order to improve its granularity. Besides a reduced cell size, the addition of timing information is helpful in the discrimination between energy deposits from the hard scattering interactions and pile-up. Improved granularity and timing will also match with the extension of tracker coverage. Many solution are under scrutiny, with the most ambitious aiming to $10 \times 10 \mathrm{~mm}^{2}$ cell readout and $10 \mathrm{ps}$ timing [6].

Similar considerations apply to the muon systems, where detector ageing and rate limitations are mainly relevant in the end-caps. Also high fake rejection at the trigger level needs to be achieved. Solutions are being elaborated based on recent progresses on Micro-Pattern Gas Detectors [7], which combine high spacial and timing resolution with high rate capability on large area detector. Significant improvements are also expected from the adoption of fast electronics in SiGe technology [8].

For the realisation of all these new detectors, one should always remind that higher rate and granularity are not free, but they put pressure on all the services needed for the operation of the detectors. Challenges are therefore not only on the detectors themselves, but smart solutions are required for power distributions, cooling and data transfer. Point-of-load and serial powering are becoming common approaches for powering the front-end electronics. Evaporative $\mathrm{CO}_{2}$ systems are now almost a standard for silicon detector operation, with active R\&D on micro-channels and light support structure to reduce the detector material. Also the development of radiation hard optical fibres and optoelectronics is a critical issue for the HL-LHC detectors.

An essential item for the full exploitation of HL-LHC is that the physics output of the trigger system cannot be degraded with respect to the one achieved at the LHC. This can only be maintained by improving on the background rejection. Trigger algorithms must therefore aim to achieve the same level of performance that is now available only after offline event processing.

One of the key ingredients to reach this goal is the inclusion of tracking information already in the first trigger levels. The details of the implementation depends on the geometry and readout architecture of the experiments [9], but a joint ATLAS and CMS R\&D effort shows a general architecture is emerging. It is schematically displayed in fig. 4: trigger processors are designed within the ATCA standard and use both custom ASICS for high speed parallel processing (associative memory chips performing pattern recognition [17]) coupled to high-end FPGAs for track fitting. The required high-density of patterns pushes towards the smallest available technology node. For example, $28 \mathrm{~nm}$ processes can provide 35\% lower power consumption for the same performance as $65 \mathrm{~nm}$ processes, and they also allow for a $4 \times$ increase in density.

The same goal of implementing trigger algorithms with thresholds and object quality as similar as possible to the offline reconstruction, is also pursued by upgrades of the trigger electronics of calorimetric and muon systems. As an example, both ATLAS and CMS are planning to have full granularity readout of the electromagnetic calorimeter. This provides a significantly better energy resolution and background rejections for electrons and photons than what can be achieved with the coarser tower readout implemented for the first phase of LHC running.

Despite these improvements, in order to keep the same physics output at the HL-LHC luminosities, the rate of events saved on disk for offline analysis will increase from the $<1 \mathrm{kHz}$ rate for the current operation to the order of $\approx 10 \mathrm{kHz}$. Together with an event size increase of approxi- 


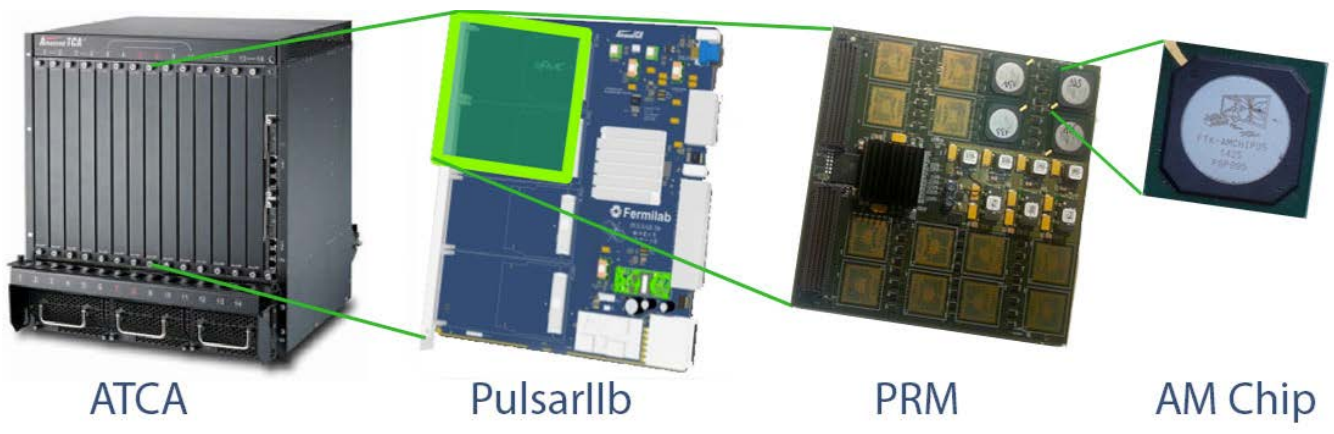

Figure 4: General architecture of a track trigger at HL-LHC, combining ATCA crate, fast FPGA and associative memory ASIC.

mately a factor 4, this will provide an enormous increase of the amount of data to be processed for offline analysis. This is putting pressure on the computing resources, since the Moore's law that predicts a doubling of computing power at constant cost every year is not valid anymore. Wide scale usage of parallel systems and high performance computing may alleviate the problem, but it is also interesting to explore other alternatives. In that perspective, it is interesting to follow the evolution of the approaches pioneered by the ALICE experiment, which saves only a fraction of the data of non-interesting events [18], and by the LHCb experiment, where the option to run triggerless and to perform online the full evet reconstruction may lead to a more refined and efficient selection of the events to be kept for offline analysis [19].

\section{Beyond HL-LHC}

While providing effective solutions to for the running at the HL-LHC is the immediate challenge, there are plenty of ideas for future experiments requiring innovative technologies. It is not possible to go through all of them, therefore only two interesting examples are reported in this presentation.

The first highlighted experimental proposal is the extreme flavour experiment mentioned in the Flavour Physics chapter of the White Paper [2]. It is "extreme" because it pushes at the limit the concept introduced at the end of the previous section, i.e. performing full event reconstruction directly during data-taking. It proposes a model in which the computing power of the online farm is used to output from the detector only the reduced set of digested physics data needed to perform the final step of the data analysis. It is a provocative proposal, but follows from the understanding that the usage of the large amount of $b$ and $c$ events produced at the HL-LHC or by a next generation flavour experiment (see section 2), requires also a rethinking of the analysis strategy and of the computing model. Maybe this proposal cannot be followed till the very end, since there is still not a strategy to perform also online calibrations and evaluate systematics uncertainties, but it is worthwhile to investigate.

Incidentally, one should notice that a requirement from such kind of high intensity experiment is to have precise time information together with high resolution position measurement in the tracking devices, in order to discriminate between overlapping events. Semiconductor detectors with a 

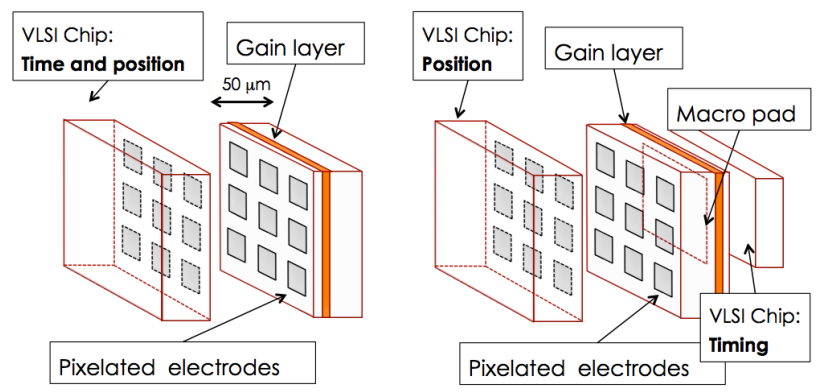

(a)

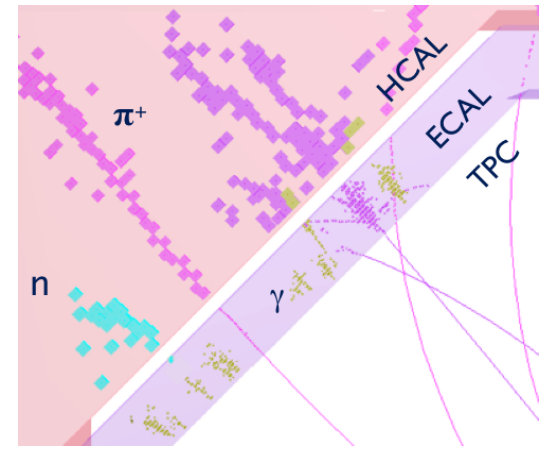

(b)

Figure 5: (a) Sketch of two possible configurations of an sensor and associated VLSI electronics: single read-out chip and split read-out.[2]. (b) A section of a typical $250 \mathrm{GeV}$ jet in ILD; the subdetectors and several particles are labelled [22].

time resolution of the order of 30 ps or less (termed Ultra Fast Silicon Detector, UFSD) are the subject of intense R\&D and may have a wide range of applications [10]. A sketch of these sensors, characterized by a thin gain layer is shows in fig. 5(a).

A second point that is worthwhile mentioning is that electron-positrons colliders are machines with well motivated physics case, capable to improve our knowledge of the Standard Model at a level unreachable at the HL-LHC and potentially able to explore also a wide range of new physics. These machines are already technically feasible [20, ?], but the overall structure of the accelerator depends on the choice of the energy range to cover. The Higgsstrahlung peak and the $t \bar{t}$ threshold region are clear targets of an $e^{+} e^{-}$program. The extension towards high energy, $\sqrt{s}=1 \mathrm{TeV}$ at a linear collider, or towards a high integrated luminosity run on the $Z$ peak and $W W$ threshold, will depend on the outcome of the LHC physics program.

Detectors at these machines are often considered "easier" than the ones at the LHC. Nevertheless it should be reminded that, to exploit at maximum the more favourable experimental environment of a lepton collider, one should aim at an experimental apparatus with the best possible resolution and uncompromising performance. For example these experiments will require very high granularity in the calorimeter, in order to perform high precision event reconstruction using particle flow algorithms as illustrated in fig. 5(b) [6, 22]. In a similar in the inner tracker, resolution should be optimised by reducing multiple scattering at a minimum. In this application monolithic pixels will be the devices of choice. There are many competing technologies: CMOS MAPS, HV- and HR-CMOS, SOI, and DEPFET. INFN has an important tradition in the design and realisation of these kinds of advanced detectors and should contribute to the shaping of these future experiments.

\section{Closing remarks}

The design and construction of the detector upgrades for the HL-LHC is the immediate challenge. For the next decade, it will keep most of the particle physics community working at accelerators focused on pushing the state-of-art of the high-rate and high-radiation hardness detectors. 
But there is a number of other interesting opportunities in the future, on precision Standard Model measurements, in the search of new physics and new particles both in the high-mass and in the low-mass "dark" sector, in testing rare or forbidden decays.

With such a vast landmark of options, within a couple of year, the results of the LHC run-2 may provide some indication about the most promising research line to follow. In the meanwhile, as recommended in the White Paper [2], an adequate effort should be put in the R\&D activities needed to provide technical solutions to the challenges the new experiments will have to face. The goal is to make these new experimental ideas to evolve into complete proposal and, hopefully, into real experiments.

Despite the ideas span very different environments and operating conditions, there are some common lines that will be worthwhile to investigate independently from the actual experiments that will be finally realised. Timing is one of the keywords found in all the systems of particle physics experiments, tracking, calorimetry and muon chambers: detectors with improved time resolution will find applications in many of the new experiments. Another common point is the interest in the trigger architecture and in moving to the online most of the reconstruction usually done offline to reduce the amount of computing resources needed for offline analyses. This workshop could be place to find synergies among the experts involved in the various INFN activities in order to boost the research on the most promising future technologies.

\section{References}

[1] ATLAS Collaboration, Observation of a new particle in the search for the Standard Model Higgs boson with the ATLAS detector at the LHC, Phys.Lett. B716 (2012) 1Đ29, arXiv: 1207.7214. CMS Collaboration, Observation of a new boson at a mass of $125 \mathrm{GeV}$ with the CMS experiment at the LHC, Phys.Lett. B716 (2012) 30Đ61, arXiv: 1207 . 7235.

[2] INFN Commissione Scientifica Nazionale 1, What Next: the White Paper of CSN1, Volume 60, Frascati Physics Series, Frascati, 2015

[3] A. Barachetti, L. Rossi and A. Szeberenyi, Final Project Report, CERN-ACC-2016-007, CERN, Geneva, 2016

[4] G. F. Della Betta, contribution to these proceedings

[5] P. Giubilato, contribution to these proceedings

[6] G. Gaudio and P. Meridiani, contribution to these proceedings

[7] S. Levorato and S. Della Torre, contribution to these proceedings

[8] R. Cardarelli and I. De Mitri, contribution to these proceedings

[9] G. Punzi, contribution to these proceedings

[10] N. Cartiglia, contribution to these proceedings

[11] D. Asner et al., ILC Higgs White Paper, arXiv:1310.0763.

[12] SHIP Collaboration, A Facility to Search for Hidden Particles (SHiP) at the CERN SPS, Technical Proposal arXiv: 1504.04956 . S. Alekhin et al., A facility to Search for Hidden Particles at the CERN SPS: the SHiP physics case, arXiV:1504.04855. 
[13] M. Raggi and V. Kozhuharov, Proposal to Search for a Dark Photon in Positron on Target Collisions at DAФNE Linac, Advances in High Energy Physics (2014), 959802 arXiv: 1306.6329

[14] Accelerating science and innovation: societal benefits of European research in Particle Physics, CERN-Brochure-2013-004-Eng, http: / / cds . cern. ch/record/1551933.

[15] ATLAS Collaboration, Letter of Intent for the Phase-II Upgrade of the ATLAS Experiment, CERN-LHCC-2012-022, CERN, Geneva, 2012 // ATLAS Collaboration, ATLAS Phase-II Upgrade Scoping Document, CERN-LHCC-2015-020, CERN, Geneva, 2015

[16] CMS Collaboration, Technical Proposal for the Phase-II Upgrade of the Compact Muon Solenoid, Technical Report CERN-LHCC-2015-010, CERN, Geneva, 2015

CMS Collaboration, CMS Phase II Upgrade Scope Document, CERN-LHCC-2015-019, CERN, Geneva, 2015

[17] ATLAS Collaboration Fast TracKer (FTK) Technical Design Report, CERN-LHCC-2013-007, CERN, Geneva, 2013

[18] M. Krzewicki, The ALICE High Level Trigger: status and plans, Journal of Physics: Conference Series 664 (2015) 082023

[19] LHCb, collaboration, LHCb Trigger and Online Upgrade Technical Design Report, CERN-LHCC-2014-016, CERN, Geneva, 2014

[20] H. Baer at al. (Editors), The International Linear Collider Technical Design Report - Volume 2: Physics, arXiv:1306.6352

T. Behnke at al. (Editors), The International Linear Collider Technical Design Report - Volume 4: Detectors, arXiv:1306.6329

[21] M. Bicer et al., First look at the physics case of TLEP, JHEP 01 (2014) 164

[22] J. S. Marshall and M. A. Thomson, The Pandora Particle Flow Algorithm, arXiv: 1308.4537 